\title{
mHealth Interventions for Health System Strengthening in China: A Systematic Review
}

Maoyi Tian ${ }^{1,2}$, MSc, PhD; Jing Zhang ${ }^{1}$, MSc; Rong Luo ${ }^{1}$, MPH; Shi Chen ${ }^{3}$, MSc; Djordje Petrovic ${ }^{4}$, MPH; Julie Redfern ${ }^{2}$, PhD; Dong Roman Xu ${ }^{5}$, MPP; Anushka Patel ${ }^{2}$, MBBS, PhD, FRACP, FCSANZ

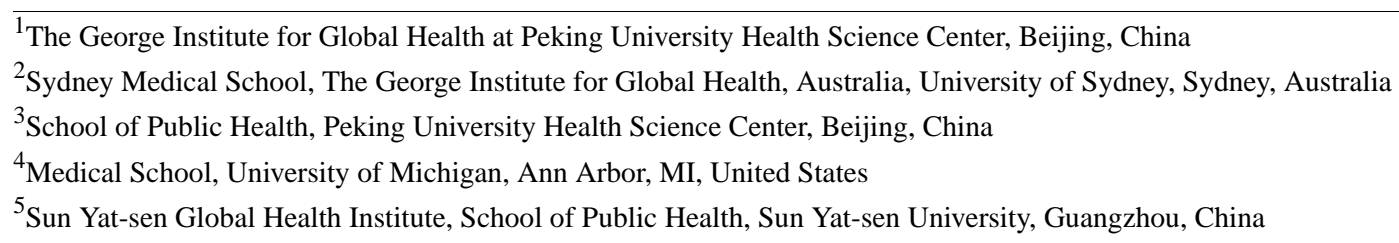

Corresponding Author:

Maoyi Tian, MSc, PhD

The George Institute for Global Health at Peking University Health Science Center

Suite 1801, Tower B, Horizon Tower

6 Zhichun Road

Beijing, 100088

China

Phone: 861082800577 ext 303

Fax: 861082800177

Email: mtian@georgeinstitute.org.cn

\section{Abstract}

Background: With rapidly expanding infrastructure in China, mobile technology has been deemed to have the potential to revolutionize health care delivery. There is particular promise for mobile health (mHealth) to positively influence health system reform and confront the new challenges of chronic diseases.

Objective: The aim of this study was to systematically review existing mHealth initiatives in China, characterize them, and examine the extent to which mHealth contributes toward the health system strengthening in China. Furthermore, we also aimed to identify gaps in mHealth development and evaluation.

Methods: We systematically reviewed the literature from English and Chinese electronic database and trial registries, including PubMed, EMBASE, Cochrane, China National Knowledge of Infrastructure (CNKI), and World Health Organization (WHO) International Clinical Trials Registry Platform. We used the English keywords of mHealth, eHealth, telemedicine, telehealth, mobile phone, cell phone, text messaging, and China, as well as their corresponding Chinese keywords. All articles using mobile technology for health care management were included in the study.

Results: A total of 1704 articles were found using the search terms, and eventually 72 were included. Overall, few high quality interventions were identified. Most interventions were found to be insufficient in scope, and their evaluation was of inadequate rigor to generate scalable solutions and provide reliable evidence of effectiveness. Most interventions focused on text messaging for consumer education and behavior change. There were a limited number of interventions that addressed health information management, health workforce issues, use of medicines and technologies, or leadership and governance from a health system perspective.

Conclusions: We provide four recommendations for future mHealth interventions in China that include the need for the development, evaluation and trials examining integrated mHealth interventions to guide the development of future mHealth interventions, target disadvantaged populations with mHealth interventions, and generate appropriate evidence for scalable and sustainable models of care.

(JMIR Mhealth Uhealth 2017;5(3):e32) doi: 10.2196/mhealth.6889

\section{KEYWORDS}

mHealth; China; health care systems 


\section{Introduction}

\section{Burden of Disease and Health System in China}

In the last decade, China has undergone a continuing epidemiological transformation from infectious diseases to chronic and noncommunicable diseases (NCDs) [1,2]. NCDs caused over $80 \%$ of China's total disability-adjusted life years in 2013 and accounted for China's largest burden of disease [3]. Chronic and NCDs pose special challenges to existing health systems as the long-term ongoing management of such conditions requires a shift from institutional care to community-based care, with an increased focus on self-management with or without peer or family support [4]. Despite the four major rounds of health care reforms since mid-1980s in China, many health equity and system level challenges remain [4,5]. Responding to those challenges, the health system needs to be adjusted to provide more effective solutions. The portability and connectivity of mobile health (mHealth) can potentially serve as an effective tool in facilitating this adjustment and to allow the health care delivery to reach hard-to-reach population. mHealth has been variably defined. The World Health Organization (WHO) definition is medical and public health practice supported by mobile devices, such as mobile phones, personal digital assistants (PDAs), and other wireless devices [6]. mHealth involves the use of a wide range of functionalities incorporated by such mobile devices, including standard voice, short message service (SMS), Web browsing, and applications on different operating systems.

\section{Chinese Mobile Market and the Potential for mHealth}

The unprecedented uptake of mobile phones with an ever growing telecommunications infrastructure has driven the development of mHealth innovation around the globe. In China, mobile phone penetration reached 94.5 per 100 people in 2014 [7]. Cellular signals now cover almost all residential areas from densely populated cities to remote villages, with increasing penetration of $3 \mathrm{G}$ and $4 \mathrm{G}$ networks. Penetration of smartphones has also increased rapidly, reaching $90 \%$ in urban areas and $32 \%$ in rural areas in 2015 [8]. The rapid development of this mobile infrastructure has created significant potential for mHealth interventions in China.

The rapid adoption of mobile phones may be explained by the diffusion of innovation theory, which is one of the most popular theories for studying adoption of information technologies and understanding how information technology innovations spread within and between communities [9].

\section{Prior Work and Objectives}

Although there were several reviews documenting the mHealth interventions in low- and middle-income countries (LMICs) [10-12], no systematic reviews of the scope and value of mHealth initiatives in the largest developing country exist. The specific aims of this systematic review were to (1) characterize mHealth interventions across all disease areas in China, (2) evaluate the extent to which mHealth interventions focus on health system strengthening, and (3) identify gaps in mHealth intervention development and evaluation that need to be addressed in the future.

\section{Methods}

\section{Database Search}

A systematic search of the literature in both Chinese and English published from May 26, 2008 to December 17, 2015, was performed following Preferred Reporting Items for Systematic Reviews and Meta-Analyses (PRISMA) guidelines [13] using the following electronic databases: PubMed, EMBASE, Cochrane, and China National Knowledge of Infrastructure (CNKI). We also searched for registered trials in the WHO International Clinical Trials Registry Platform, which included 15 approved trial registries and supplementary searches in Chinese Clinical Trial Registry (CHICTR), and Clinicaltrials.gov. English keywords used in these searches included the following: mHealth, eHealth, telemedicine, telehealth, mobile phone, cell phone, text messaging, and China. The Chinese keywords used include "ShouJi" (mobile phone or cell phone), "DuanXin" (text messaging), "YiDongJiangKang" (mHealth), and "Yi Dong Yi Liao" (mobile medicine). Multimedia Appendix 1 lists the detailed search strategy for each database.

\section{Inclusion and Exclusion Criteria}

We included all articles related to health care management using mobile technology in China. Any type of the following articles with full texts was included: (1) randomized controlled trials (RCTs), (2) quasi-experimental studies, (3) descriptive studies without any outcome measured, or (4) registered RCTs. We only included studies written in English or Chinese, and articles related to telemedicine or telehealth were only included if mobile technologies were used as part of the intervention. We excluded all articles describing technology development, review articles, protocol papers, and any studies using fixed landline phone or the Internet using a desktop computer as part of the intervention. A total of 5 reviewers independently evaluated and excluded articles at the abstract review stage. Full-text articles whose abstracts met the inclusion criteria were then reviewed by 3 reviewers.

\section{Analytical Framework}

We utilized an adapted health system framework to evaluate the role of mHealth interventions as a health system strengthening tool (Figure 1) [14-16]. In this framework, there were two dimensions: (1) the function of mHealth intervention categorizing into one of the $12 \mathrm{mHealth}$ tools proposed by Labrique et al [14], and (2) the corresponded health system frame work as developed by Hsiao and WHO [15,16]. Assessing both dimensions of the mHealth intervention allowed us to identify where the gaps were in the mHealth interventions from a health systems perspective. 
Figure 1. Adapted health system framework for evaluating mHealth interventions.

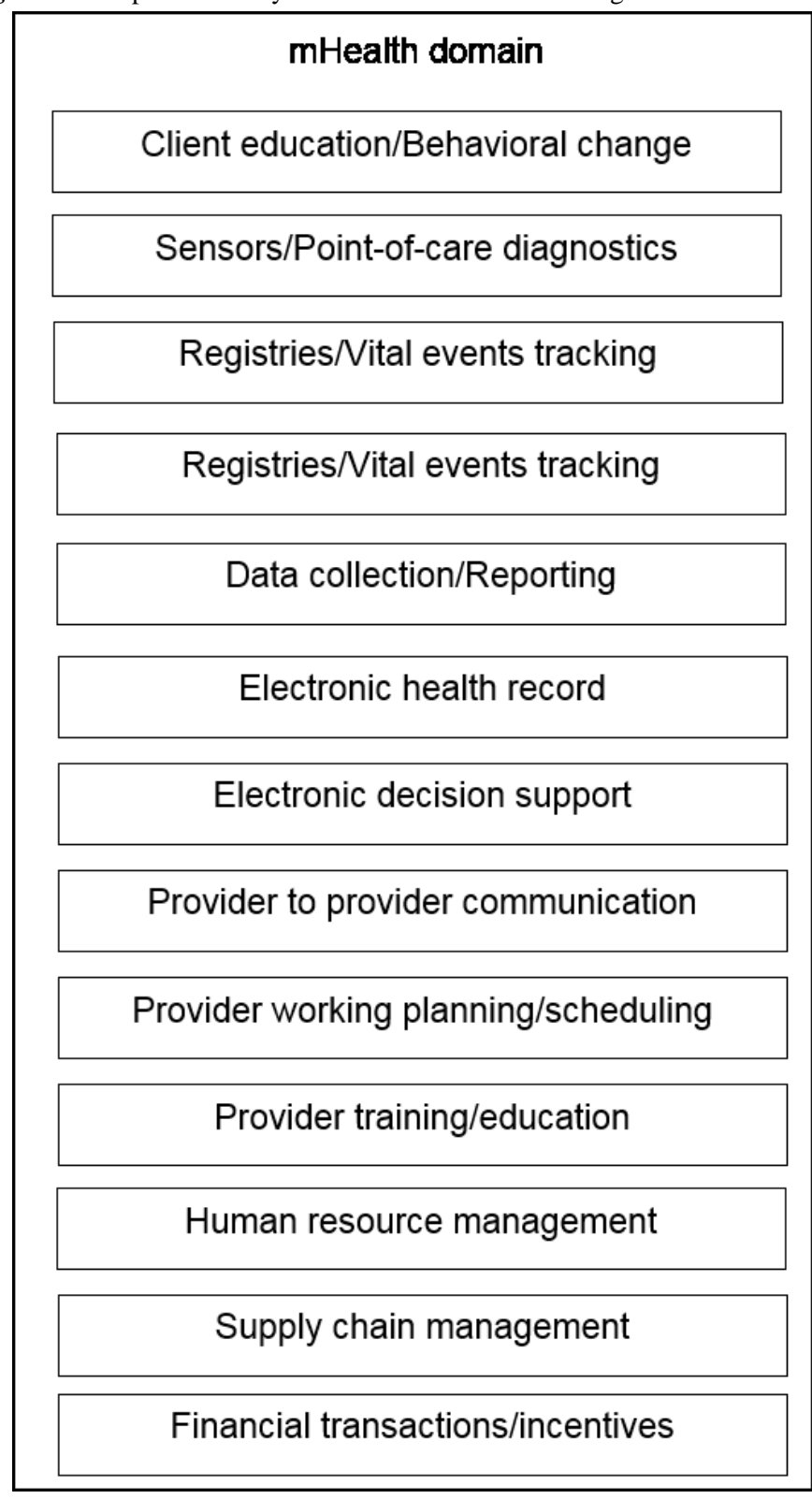

\section{Data Extraction}

A spreadsheet was developed for entering extracted data that included study characteristics, the mHealth domain, and the health system domain using the aforementioned analytical framework [16]. An agreement was reached on the definitions and interpretation of each variable in the data extraction template among the reviewers before data collection. Three reviewers independently extracted the data into the template and cross-reviewed. Disagreements in this step were resolved by consensus.

\section{Quality Assessment}

For RCTs, methodological quality was assessed using the Cochrane Risk of Bias Assessment Tool [17]. We assessed the

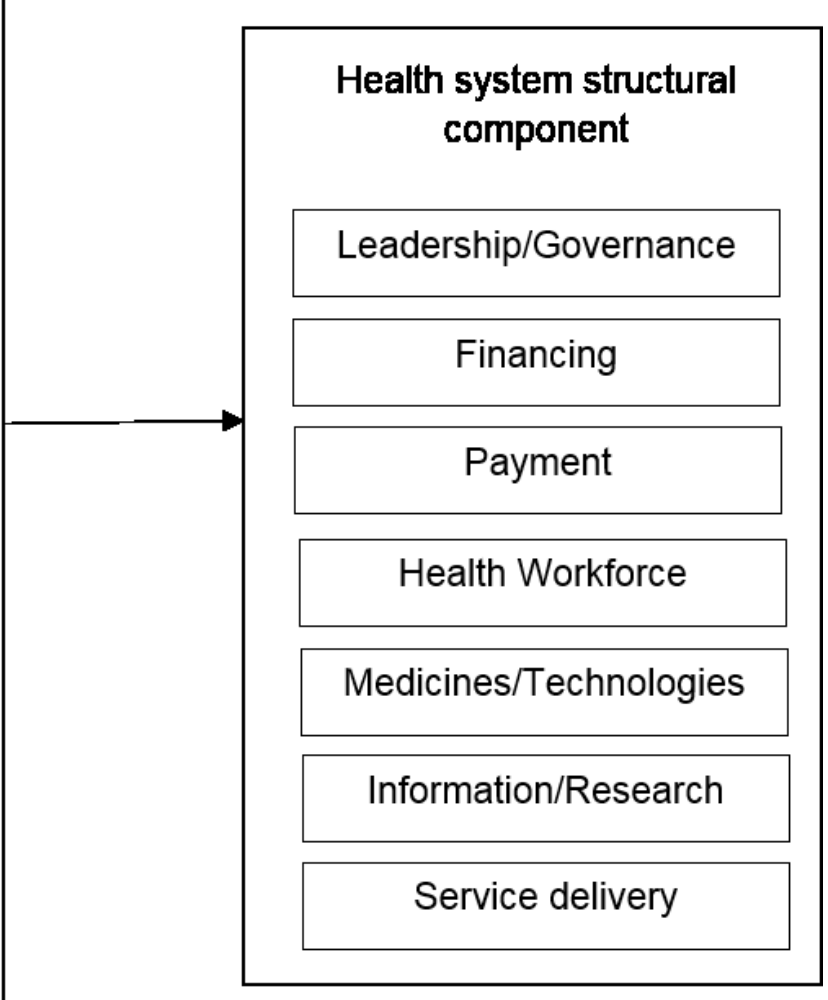

random sequence generation, allocation concealment, blinding of participants, personnel and outcome assessors, incomplete outcome data, selective outcome reporting, and other sources of bias. Any discrepancies in article inclusion, data extraction, and bias assessment were discussed and resolved by team consensus.

\section{Results}

\section{Included Studies}

We retrieved 1704 articles using the search terms, and 323 articles were selected for full-text review (Figure 2). Of those, 251 studies were excluded for the following reasons: not conducted in China $(n=81)$, not using the mobile technology $(n=142)$, protocol papers $(n=6)$, and review articles $(n=22)$. 
Figure 2. Study flowchart.

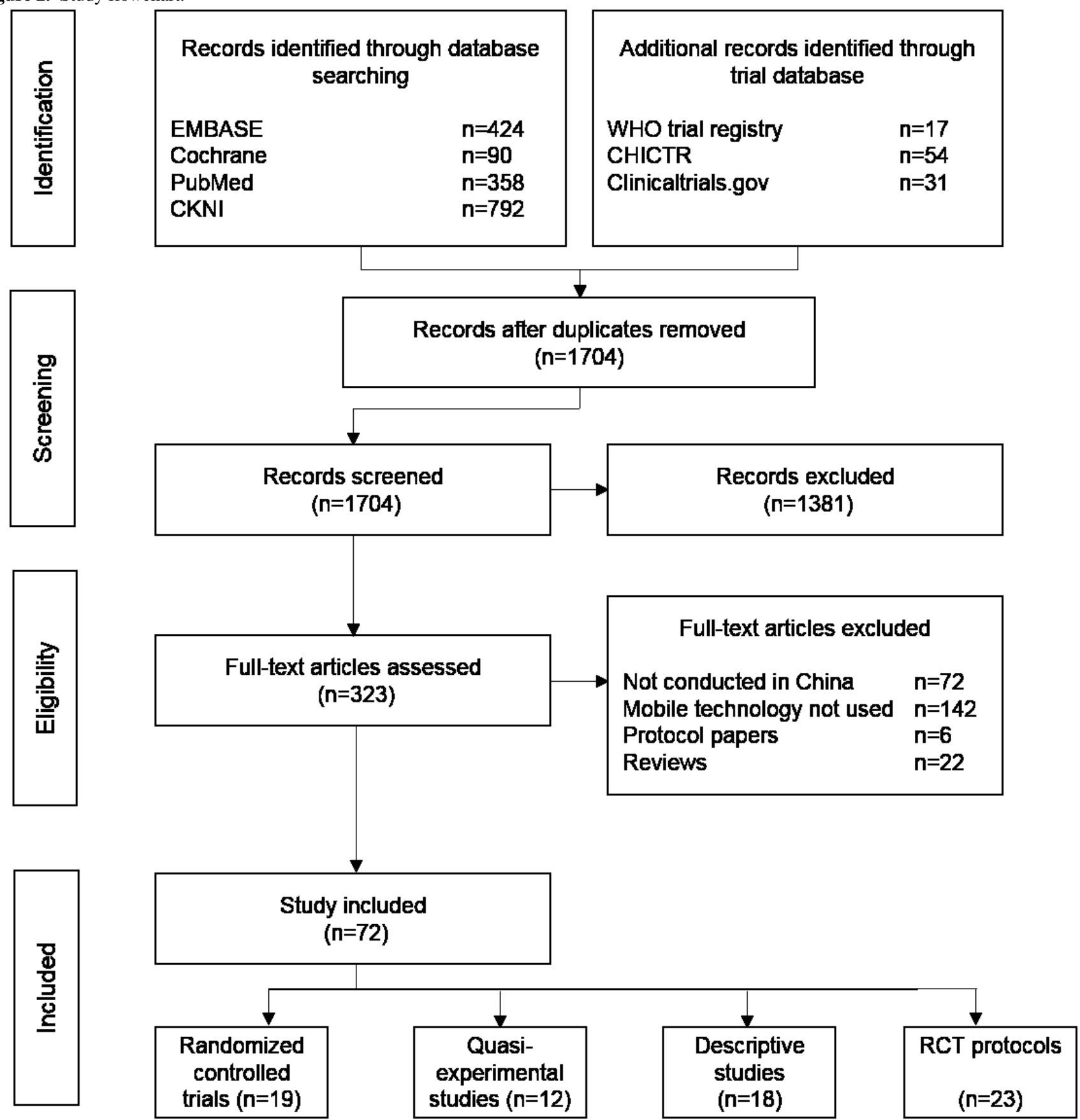

\section{Study Characteristics}

The study characteristics, mHealth domain, and health system domain of the nonprotocol articles $(n=49)$ are summarized in Table 1 . The majority of the studies were conducted in an urban setting $(n=34)$ [18-51], with only 6 focusing on a rural population [28,51-55]. The most common disease focus was on NCDs $(n=15) \quad[22,25,26,29,30,34,37-39,42,45,46,52,56,57]$, whereas 12 studies focused on infectious diseases $[33,41,51,53,54,58-64]$ and 8 studies were designed for maternal and child health $[36,40,43,47-49,55,65]$. A wide range of study designs was used to evaluate or describe the mHealth intervention, including 18 exploratory studies that described, validated, or pilot-tested mHealth interventions without any quantitative outcome assessment [18-28,58-62,64,66]. A total of 31 studies quantitatively evaluated the mHealth intervention [29-57,63,65], of which 19 utilized a RCT design [29-35,38-40,43,47,51-53,56,57,63,65] whereas the remainder used a quasi-experimental study design $(n=12)$. In most cases, the primary mobile technology was a regular mobile phone $(n=36)[18,19,21,25,29-49,51,53-57,59,62-65]$. Only 12 studies utilized smartphone technology for the intervention [20,22-24,26-28,50,52,58,61,66]. 
Table 1. Study characteristics, mHealth domain, and health system domain of nonprotocol articles.

\begin{tabular}{|c|c|c|c|c|c|c|c|}
\hline Author & Setting & Disease area & $\begin{array}{l}\text { Population } \\
\text { (n) }\end{array}$ & Study description & $\begin{array}{l}\text { Type of } \\
\text { device }\end{array}$ & $\begin{array}{l}\text { mHealth } \\
\text { domain }\end{array}$ & $\begin{array}{l}\text { Health system } \\
\text { domain }\end{array}$ \\
\hline \multicolumn{8}{|c|}{ Descriptive Studies } \\
\hline Deng [18] & Urban & $\begin{array}{l}\text { Others } \\
\text {-patients for sedation } \\
\text { gastrointestinal en- } \\
\text { doscopy (SGIE) }\end{array}$ & $\begin{array}{l}908 \text { outpa- } \\
\text { tients in the } \\
\text { anesthesia } \\
\text { clinic for } \\
\text { SGIE }\end{array}$ & $\begin{array}{l}\text { Feasibility to use SMS } \\
\text { to improve the adher- } \\
\text { ence for SGIE appoint- } \\
\text { ment }\end{array}$ & $\mathrm{R}^{\wedge}$ & $\begin{array}{l}\text { Client educa- } \\
\text { tion and behav- } \\
\text { ior change }\end{array}$ & Service delivery \\
\hline Chen [19] & Urban & $\begin{array}{l}\text { Others } \\
\text {-suicide } \\
\text { attempters }\end{array}$ & $\begin{array}{l}15 \text { suicide at- } \\
\text { tempters from } \\
\text { the emergency } \\
\text { department }\end{array}$ & $\begin{array}{l}\text { Feasibility to SMS to } \\
\text { decrease recidivism for } \\
\text { suicide attempters }\end{array}$ & $\mathrm{R}$ & $\begin{array}{l}\text { Client educa- } \\
\text { tion and behav- } \\
\text { ior change }\end{array}$ & Service delivery \\
\hline $\operatorname{Li}[58]$ & $\begin{array}{l}\text { Not } \\
\text { described }\end{array}$ & Infectious disease & $\begin{array}{l}\text { Not } \\
\text { described }\end{array}$ & $\begin{array}{l}\text { A decision support sys- } \\
\text { tem for the responses to } \\
\text { infectious disease } \\
\text { emergencies }\end{array}$ & $\mathrm{S}^{*}$ & $\begin{array}{l}\text { Electronic de- } \\
\text { cision support }\end{array}$ & $\begin{array}{l}\text { Leadership/gov- } \\
\text { ernance }\end{array}$ \\
\hline Zhao [20] & Urban & $\begin{array}{l}\text { Not } \\
\text { mentioned }\end{array}$ & $\begin{array}{l}\text { Not } \\
\text { described }\end{array}$ & $\begin{array}{l}\text { A case report describ- } \\
\text { ing development of a } \\
\text { shared community } \\
\text { health information sys- } \\
\text { tem }\end{array}$ & $\mathrm{S}$ & $\begin{array}{l}\text { Electronic } \\
\text { medical } \\
\text { record }\end{array}$ & $\begin{array}{l}\text { Leadership/gov- } \\
\text { ernance }\end{array}$ \\
\hline $\operatorname{Li}[59]$ & $\begin{array}{l}\text { Not } \\
\text { described }\end{array}$ & $\begin{array}{l}\text { Infectious disease } \\
\text {-hand, foot, and } \\
\text { mouth disease }\end{array}$ & $\begin{array}{l}\text { Not } \\
\text { described }\end{array}$ & $\begin{array}{l}\text { Use of SMS to develop } \\
\text { automated alert and re- } \\
\text { sponse system for hand, } \\
\text { foot, and mouth disease }\end{array}$ & $\mathrm{R}$ & $\begin{array}{l}\text { Registries and } \\
\text { vital event } \\
\text { tracking }\end{array}$ & $\begin{array}{l}\text { Leadership/gov- } \\
\text { ernance }\end{array}$ \\
\hline Guo [60] & $\begin{array}{l}\text { Not } \\
\text { described }\end{array}$ & Infectious disease & $\begin{array}{l}\text { Not } \\
\text { described }\end{array}$ & $\begin{array}{l}\text { A mobile phone-based } \\
\text { infectious disease report- } \\
\text { ing system in earth- } \\
\text { quake-affected area }\end{array}$ & $\mathrm{PDA}^{\mathrm{a}}$ & $\begin{array}{l}\text { Data collec- } \\
\text { tion and report- } \\
\text { ing }\end{array}$ & Information \\
\hline Mao [21] & Urban & $\begin{array}{l}\text { Not } \\
\text { mentioned }\end{array}$ & $\begin{array}{l}100 \text { patients } \\
\text { admitted from } \\
\text { general hospi- } \\
\text { tal }\end{array}$ & $\begin{array}{l}\text { Use of SMS to deliver } \\
\text { individualized pharma- } \\
\text { ceutical care }\end{array}$ & $\mathrm{R}$ & $\begin{array}{l}\text { Client educa- } \\
\text { tion and behav- } \\
\text { ior change }\end{array}$ & Service delivery \\
\hline Yang [61] & $\begin{array}{l}\text { Not } \\
\text { described }\end{array}$ & Infectious disease & $\begin{array}{l}495 \text { health } \\
\text { care agencies } \\
\text { in earthquake- } \\
\text { affected area }\end{array}$ & $\begin{array}{l}\text { Use of mobile phone as } \\
\text { a surveillance tool to } \\
\text { monitor infectious dis- } \\
\text { ease }\end{array}$ & $\mathrm{S}$ & $\begin{array}{l}\text { Data collec- } \\
\text { tion and report- } \\
\text { ing }\end{array}$ & Information \\
\hline Jun [22] & Urban & $\begin{array}{l}\text { Noncommunicable } \\
\text { disease } \\
\text {-adolescent Idiopath- } \\
\text { ic Scoliosis }\end{array}$ & $\begin{array}{l}64 \text { adolescent } \\
\text { idiopathic sco- } \\
\text { liosis patients }\end{array}$ & $\begin{array}{l}\text { Use of smartphone to } \\
\text { measure the axial trunk } \\
\text { rotation }\end{array}$ & $\mathrm{S}$ & $\begin{array}{l}\text { Sensors and } \\
\text { point-of-care } \\
\text { diagnosis }\end{array}$ & $\begin{array}{l}\text { Medicines/tech- } \\
\text { nologies }\end{array}$ \\
\hline Zhang [64] & $\begin{array}{l}\text { Not } \\
\text { described }\end{array}$ & $\begin{array}{l}\text { Infectious disease } \\
\text {-schistoscomajapon- } \\
\text { icum infection }\end{array}$ & $\begin{array}{l}\text { Not } \\
\text { described }\end{array}$ & $\begin{array}{l}\text { Use of SMS to send } \\
\text { alert the fishermen to } \\
\text { avoid the schistosome } \\
\text { infection }\end{array}$ & $\mathrm{R}$ & $\begin{array}{l}\text { Registries and } \\
\text { vital event } \\
\text { tracking }\end{array}$ & $\begin{array}{l}\text { Leadership/gov- } \\
\text { ernance }\end{array}$ \\
\hline $\mathrm{Ma}[62]$ & $\begin{array}{l}\text { Not } \\
\text { described }\end{array}$ & Infectious disease & $\begin{array}{l}\text { Not } \\
\text { described }\end{array}$ & $\begin{array}{l}\text { Development of SMS- } \\
\text { based emergency re- } \\
\text { sponse system for infec- } \\
\text { tious disease }\end{array}$ & $\mathrm{R}$ & $\begin{array}{l}\text { Registries and } \\
\text { vital event } \\
\text { tracking }\end{array}$ & $\begin{array}{l}\text { Leadership/gov- } \\
\text { ernance }\end{array}$ \\
\hline Guan [23] & Urban & $\begin{array}{l}\text { Others } \\
\text {-voiding diary moni- } \\
\text { toring }\end{array}$ & $\begin{array}{l}20 \text { healthy } \\
\text { volunteers }\end{array}$ & $\begin{array}{l}\text { Development of smart- } \\
\text { phone-based remote } \\
\text { voiding diary monitor- } \\
\text { ing system }\end{array}$ & $\mathrm{S}$ & $\begin{array}{l}\text { Data collec- } \\
\text { tion and report- } \\
\text { ing }\end{array}$ & Service delivery \\
\hline Ye [24] & Urban & $\begin{array}{l}\text { Others } \\
\text {-slitlampbiomi- } \\
\text { croscopy }\end{array}$ & $\begin{array}{l}\text { Not } \\
\text { described }\end{array}$ & $\begin{array}{l}\text { Use of smartphone } \\
\text { camera for teleophthal- } \\
\text { mology }\end{array}$ & $\mathrm{S}$ & $\begin{array}{l}\text { Sensors and } \\
\text { point-of-care } \\
\text { diagnosis }\end{array}$ & Service delivery \\
\hline
\end{tabular}




\begin{tabular}{|c|c|c|c|c|c|c|c|c|}
\hline Author & Setting & Disease area & $\begin{array}{l}\text { Population } \\
\text { (n) }\end{array}$ & Study description & & $\begin{array}{l}\text { Type of } \\
\text { device }\end{array}$ & $\begin{array}{l}\text { mHealth } \\
\text { domain }\end{array}$ & $\begin{array}{l}\text { Health system } \\
\text { domain }\end{array}$ \\
\hline Yu [66] & $\begin{array}{l}\text { Not } \\
\text { described }\end{array}$ & $\begin{array}{l}\text { Not } \\
\text { mentioned }\end{array}$ & 11 volunteers & $\begin{array}{l}\text { Health examination } \\
\text { toolkit involving sen- } \\
\text { sors and data upload in- } \\
\text { to an Android phone }\end{array}$ & & $\mathrm{S}$ & $\begin{array}{l}\text { Sensors and } \\
\text { point-of-care } \\
\text { diagnosis }\end{array}$ & Service delivery \\
\hline Yin [25] & Urban & $\begin{array}{l}\text { Noncommunicable } \\
\text { disease } \\
\text {-dialysis patients }\end{array}$ & $\begin{array}{l}\text { Not } \\
\text { described }\end{array}$ & $\begin{array}{l}\text { Development of mobile } \\
\text { phone-based follow up } \\
\text { system }\end{array}$ & & $\mathrm{R}$ & $\begin{array}{l}\text { Client educa- } \\
\text { tion and behav- } \\
\text { ior change }\end{array}$ & Service delivery \\
\hline Yang [65] & Urban & $\begin{array}{l}\text { Noncommunicable } \\
\text { disease } \\
\text {-facial acne }\end{array}$ & $\begin{array}{l}80 \text { patients } \\
\text { with facial ac- } \\
\text { ne }\end{array}$ & $\begin{array}{l}\text { Use of mobile phone to } \\
\text { grade the severity of fa- } \\
\text { cial acne }\end{array}$ & & $\mathrm{S}$ & $\begin{array}{l}\text { Sensors and } \\
\text { point-of-care } \\
\text { diagnosis }\end{array}$ & Service delivery \\
\hline Wang [27] & Urban & $\begin{array}{l}\text { Others } \\
\text {-dietary intake as- } \\
\text { sessment }\end{array}$ & $\begin{array}{l}35 \text { healthy } \\
\text { volunteers }\end{array}$ & $\begin{array}{l}\text { Development of dietary } \\
\text { intake assessment using } \\
\text { mobile phone camera } \\
\text { function }\end{array}$ & & $\mathrm{S}$ & $\begin{array}{l}\text { Data collec- } \\
\text { tion and report- } \\
\text { ing }\end{array}$ & $\begin{array}{l}\text { Medicines/tech- } \\
\text { nologies }\end{array}$ \\
\hline Smith [28] & $\begin{array}{l}\text { Rural and } \\
\text { urban }\end{array}$ & $\begin{array}{l}\text { Not } \\
\text { mentioned }\end{array}$ & $\begin{array}{l}110 \text { healthy } \\
\text { adults }\end{array}$ & $\begin{array}{l}\text { Development of a } \\
\text { smartphone-assisted 24- } \\
\text { h recall to assess bever- } \\
\text { age consumption }\end{array}$ & & $\mathrm{S}$ & $\begin{array}{l}\text { Data collec- } \\
\text { tion and report- } \\
\text { ing }\end{array}$ & $\begin{array}{l}\text { Medicines/tech- } \\
\text { nologies }\end{array}$ \\
\hline RCT & & & & Intervention & Follow-up & & & \\
\hline Tian [52] & Rural & $\begin{array}{l}\text { Noncommunicable } \\
\text { disease } \\
\text {-cardiovascular dis- } \\
\text { ease }\end{array}$ & $\begin{array}{l}2086 \text { high car- } \\
\text { diovascular } \\
\text { risk patients }\end{array}$ & $\begin{array}{l}\text { A smartphone based } \\
\text { electronic decision sup- } \\
\text { port system focusing on } \\
\text { two medication use and } \\
\text { two lifestyle modifica- } \\
\text { tions }\end{array}$ & 12 month & $\mathrm{S}$ & $\begin{array}{l}\text { Electronic de- } \\
\text { cision support }\end{array}$ & Service delivery \\
\hline Lin [29] & Urban & $\begin{array}{l}\text { Noncommunicable } \\
\text { disease } \\
\text {-obesity }\end{array}$ & $\begin{array}{l}123 \text { over- } \\
\text { weight adults }\end{array}$ & $\begin{array}{l}\text { SMS-assisted lifestyle } \\
\text { weight loss intervention }\end{array}$ & 6 month & $\mathrm{R}$ & $\begin{array}{l}\text { Client educa- } \\
\text { tion and behav- } \\
\text { ior change }\end{array}$ & Service delivery \\
\hline Liu [51] & $\begin{array}{l}\text { Rural and } \\
\text { urban }\end{array}$ & $\begin{array}{l}\text { Infectious disease } \\
\text {-tuberculosis }\end{array}$ & $\begin{array}{l}4173 \text { pul- } \\
\text { monary } \mathrm{TB}^{\mathrm{b}} \\
\text { patients }\end{array}$ & $\begin{array}{l}\text { SMS reminders and } \\
\text { medication monitoring }\end{array}$ & 6 month & $\mathrm{R}$ & $\begin{array}{l}\text { Client educa- } \\
\text { tion and behav- } \\
\text { ior change }\end{array}$ & Service delivery \\
\hline Sabin [63] & $\begin{array}{l}\text { Not } \\
\text { described }\end{array}$ & $\begin{array}{l}\text { Infectious disease } \\
-\mathrm{HIV}^{\mathrm{c}}\end{array}$ & $\begin{array}{l}120 \text { HIV pa- } \\
\text { tients }\end{array}$ & $\begin{array}{l}\text { Real time SMS re- } \\
\text { minders triggered by } \\
\text { the electronic medica- } \\
\text { tion storage device }\end{array}$ & 6 month & $\mathrm{R}$ & $\begin{array}{l}\text { Client educa- } \\
\text { tion and behav- } \\
\text { ior change }\end{array}$ & Service delivery \\
\hline Liu [30] & Urban & $\begin{array}{l}\text { Noncommunicable } \\
\text { disease } \\
\text {-cardiovascular dis- } \\
\text { ease }\end{array}$ & $\begin{array}{l}589 \text { workers } \\
\text { without } \\
\text { known } \mathrm{CVD}^{\mathrm{d}}\end{array}$ & $\begin{array}{l}\text { Mobile-phone based } \\
\text { lifestyle intervention }\end{array}$ & 12 month & $\mathrm{R}$ & $\begin{array}{l}\text { Client educa- } \\
\text { tion and behav- } \\
\text { ior change }\end{array}$ & Service delivery \\
\hline Shi [31] & Urban & $\begin{array}{l}\text { Others } \\
\text {-smokers }\end{array}$ & $\begin{array}{l}179 \text { adoles- } \\
\text { cent smokers }\end{array}$ & $\begin{array}{l}\text { Smoking cessation } \\
\text { lifestyle intervention } \\
\text { delivered by the SMS }\end{array}$ & 12 week & $\mathrm{R}$ & $\begin{array}{l}\text { Client educa- } \\
\text { tion and behav- } \\
\text { ior change }\end{array}$ & Service delivery \\
\hline Chen [53] & Rural & $\begin{array}{l}\text { Infectious disease } \\
\text {-Viral infections af- } \\
\text { fecting upper respira- } \\
\text { tory tract and otitis } \\
\text { media }\end{array}$ & $\begin{array}{l}977 \text { township } \\
\text { level health } \\
\text { workers }\end{array}$ & $\begin{array}{l}\text { SMS based health } \\
\text { worker training }\end{array}$ & 1 month & $\mathrm{R}$ & $\begin{array}{l}\text { Provider train- } \\
\text { ing and educa- } \\
\text { tion }\end{array}$ & $\begin{array}{l}\text { Health work- } \\
\text { force }\end{array}$ \\
\hline Deng [32] & Urban & $\begin{array}{l}\text { Others } \\
\text {-outpatients for seda- } \\
\text { tion gastrointestinal } \\
\text { endoscopy }\end{array}$ & $\begin{array}{l}2200 \text { outpa- } \\
\text { tients }\end{array}$ & $\begin{array}{l}\text { SMS reminders to at- } \\
\text { tend medical examina- } \\
\text { tion }\end{array}$ & $\begin{array}{l}\text { Not } \\
\text { mentioned }\end{array}$ & $\mathrm{R}$ & $\begin{array}{l}\text { Client educa- } \\
\text { tion and behav- } \\
\text { ior change }\end{array}$ & Service delivery \\
\hline $\operatorname{Lv}[56]$ & $\begin{array}{l}\text { Not } \\
\text { described }\end{array}$ & $\begin{array}{l}\text { Noncommunicable } \\
\text { disease } \\
\text {-asthma }\end{array}$ & $\begin{array}{l}150 \text { outpa- } \\
\text { tients with } \\
\text { asthma }\end{array}$ & $\begin{array}{l}\text { SMS reminders for } \\
\text { asthma self-manage- } \\
\text { ment }\end{array}$ & 12 week & $\mathrm{R}$ & $\begin{array}{l}\text { Client educa- } \\
\text { tion and behav- } \\
\text { ior change }\end{array}$ & Service delivery \\
\hline
\end{tabular}




\begin{tabular}{|c|c|c|c|c|c|c|c|c|}
\hline Author & Setting & Disease area & $\begin{array}{l}\text { Population } \\
\text { (n) }\end{array}$ & Study description & & $\begin{array}{l}\text { Type of } \\
\text { device }\end{array}$ & $\begin{array}{l}\text { mHealth } \\
\text { domain }\end{array}$ & $\begin{array}{l}\text { Health system } \\
\text { domain }\end{array}$ \\
\hline Wang [57] & $\begin{array}{l}\text { Not } \\
\text { described }\end{array}$ & $\begin{array}{l}\text { Noncommunicable } \\
\text { disease } \\
\text {-allergic rhinitis }\end{array}$ & $\begin{array}{l}50 \text { outpatients } \\
\text { with allergic } \\
\text { rhinitis }\end{array}$ & $\begin{array}{l}\text { SMS reminders to im- } \\
\text { prove adherence to } \\
\text { medication and treat- } \\
\text { ment }\end{array}$ & 30 days & $\mathrm{R}$ & $\begin{array}{l}\text { Client educa- } \\
\text { tion and behav- } \\
\text { ior change }\end{array}$ & Service delivery \\
\hline Chai [33] & Urban & $\begin{array}{l}\text { Infectious disease } \\
-\mathrm{H} 1 \mathrm{~N} 1\end{array}$ & $\begin{array}{l}1992 \text { residents } \\
\text { in Shanghai }\end{array}$ & $\begin{array}{l}\text { SMS-based health edu- } \\
\text { cation for H1N1 preven- } \\
\text { tion }\end{array}$ & 10 days & $\mathrm{R}$ & $\begin{array}{l}\text { Client educa- } \\
\text { tion and behav- } \\
\text { ior change }\end{array}$ & Service delivery \\
\hline $\operatorname{Lin}[65]$ & $\begin{array}{l}\text { Not } \\
\text { described }\end{array}$ & $\begin{array}{l}\text { Maternal and child } \\
\text { health }\end{array}$ & $\begin{array}{l}258 \text { parent- } \\
\text { child pairs } \\
\text { with child } \\
\text { having } \\
\text { cataract }\end{array}$ & $\begin{array}{l}\text { SMS reminders to at- } \\
\text { tend medical appoint- } \\
\text { ment }\end{array}$ & 4 days & $\mathrm{R}$ & $\begin{array}{l}\text { Client educa- } \\
\text { tion and behav- } \\
\text { ior change }\end{array}$ & Service delivery \\
\hline Dai [34] & Urban & $\begin{array}{l}\text { Noncommunicable } \\
\text { disease } \\
\text {-diabetes }\end{array}$ & $\begin{array}{l}80 \text { type- } 2 \text { dia- } \\
\text { betes patients }\end{array}$ & $\begin{array}{l}\text { SMS based health edu- } \\
\text { cation }\end{array}$ & 12 month & $\mathrm{R}$ & $\begin{array}{l}\text { Client educa- } \\
\text { tion and behav- } \\
\text { ior change }\end{array}$ & Service delivery \\
\hline Shi [35] & Urban & $\begin{array}{l}\text { Others } \\
\text {-smokers }\end{array}$ & $\begin{array}{l}176 \text { adoles- } \\
\text { cent smokers }\end{array}$ & $\begin{array}{l}\text { SMS based health edu- } \\
\text { cation for smoking ces- } \\
\text { sation }\end{array}$ & 3 month & $\mathrm{R}$ & $\begin{array}{l}\text { Client educa- } \\
\text { tion and behav- } \\
\text { ior change }\end{array}$ & Service delivery \\
\hline Zhang [40] & Urban & $\begin{array}{l}\text { Maternal and child } \\
\text { health }\end{array}$ & $\begin{array}{l}166 \text { children } \\
\text { with asthma }\end{array}$ & $\begin{array}{l}\text { SMS-based health pro- } \\
\text { motion }\end{array}$ & 3 month & $\mathrm{R}$ & $\begin{array}{l}\text { Client educa- } \\
\text { tion and behav- } \\
\text { ior change }\end{array}$ & Service delivery \\
\hline Wei [38] & Urban & $\begin{array}{l}\text { Noncommunicable } \\
\text { disease } \\
\text {-chronic kidney dis- } \\
\text { ease }\end{array}$ & $\begin{array}{l}108 \text { patients } \\
\text { with chronic } \\
\text { kidney disease }\end{array}$ & $\begin{array}{l}\text { SMS-based medication } \\
\text { adherence intervention }\end{array}$ & 3 month & $\mathrm{R}$ & $\begin{array}{l}\text { Client educa- } \\
\text { tion and behav- } \\
\text { ior change }\end{array}$ & Service delivery \\
\hline $\mathrm{Li}[43]$ & Urban & $\begin{array}{l}\text { Maternal and child } \\
\text { health }\end{array}$ & $\begin{array}{l}82 \text { pregnant } \\
\text { women }\end{array}$ & $\begin{array}{l}\text { SMS-based dietary rec- } \\
\text { ommendation during } \\
\text { pregnancy }\end{array}$ & $\begin{array}{l}\text { Not } \\
\text { mentioned }\end{array}$ & $\mathrm{R}$ & $\begin{array}{l}\text { Client educa- } \\
\text { tion and behav- } \\
\text { ior change }\end{array}$ & Service delivery \\
\hline Chen [74] & Urban & $\begin{array}{l}\text { Maternal and child } \\
\text { health }\end{array}$ & $\begin{array}{l}155 \text { pregnant } \\
\text { women }\end{array}$ & $\begin{array}{l}\text { SMS-based breastfeed- } \\
\text { ing promotion }\end{array}$ & 16 week & $\mathrm{R}$ & $\begin{array}{l}\text { Client educa- } \\
\text { tion and behav- } \\
\text { ior change }\end{array}$ & Service delivery \\
\hline $\mathrm{Qu}[25]$ & Urban & $\begin{array}{l}\text { Noncommunicable } \\
\text { disease } \\
\text {-schizophrenia }\end{array}$ & $\begin{array}{l}178 \text { patients } \\
\text { with } \\
\text { schizophrenia }\end{array}$ & $\begin{array}{l}\text { SMS-based medication } \\
\text { adherence intervention }\end{array}$ & 12 month & $\mathrm{R}$ & $\begin{array}{l}\text { Client educa- } \\
\text { tion and behav- } \\
\text { ior change }\end{array}$ & Service delivery \\
\hline \multicolumn{9}{|c|}{ Quasi-experiment } \\
\hline Jiang [49] & Urban & $\begin{array}{l}\text { Maternal and child } \\
\text { health }\end{array}$ & $\begin{array}{l}582 \text { expectant } \\
\text { mothers }\end{array}$ & $\begin{array}{l}\text { SMS-based intervention } \\
\text { about infant feeding }\end{array}$ & 12 month & $\mathrm{R}$ & $\begin{array}{l}\text { Client educa- } \\
\text { tion and behav- } \\
\text { ior change }\end{array}$ & Service delivery \\
\hline Fang [42] & Urban & $\begin{array}{l}\text { Noncommunicable } \\
\text { disease } \\
\text {-hypertension }\end{array}$ & $\begin{array}{l}599 \text { hyperten- } \\
\text { sive patients }\end{array}$ & $\begin{array}{l}\text { SMS-based health edu- } \\
\text { cation for hypertension } \\
\text { management }\end{array}$ & 12 month & $\mathrm{R}$ & $\begin{array}{l}\text { Client educa- } \\
\text { tion and behav- } \\
\text { ior change }\end{array}$ & Service delivery \\
\hline Zhao [46] & Urban & $\begin{array}{l}\text { Noncommunicable } \\
\text { disease } \\
\text {-diabetes }\end{array}$ & $\begin{array}{l}64 \text { type- } 2 \text { dia- } \\
\text { betes patients }\end{array}$ & $\begin{array}{l}\text { SMS-based medication } \\
\text { adherence and health } \\
\text { education program }\end{array}$ & 3 month & $\mathrm{R}$ & $\begin{array}{l}\text { Client educa- } \\
\text { tion and behav- } \\
\text { ior change }\end{array}$ & Service delivery \\
\hline Qin [44] & Urban & $\begin{array}{l}\text { Others } \\
\text {-dialysis }\end{array}$ & $\begin{array}{l}92 \text { dialysis pa- } \\
\text { tients }\end{array}$ & $\begin{array}{l}\text { SMS-based health edu- } \\
\text { cation for dialysis pa- } \\
\text { tients delivered by the } \\
\text { nurse }\end{array}$ & $\begin{array}{l}\text { 53-612 } \\
\text { days }\end{array}$ & $\mathrm{R}$ & $\begin{array}{l}\text { Client educa- } \\
\text { tion and behav- } \\
\text { ior change }\end{array}$ & Service delivery \\
\hline Xie [45] & Urban & $\begin{array}{l}\text { Noncommunicable } \\
\text { disease } \\
\text {-diabetes }\end{array}$ & $\begin{array}{l}196 \text { type- } 2 \text { dia- } \\
\text { betes patients }\end{array}$ & $\begin{array}{l}\text { SMS-based health pro- } \\
\text { motion for diabetes } \\
\text { management }\end{array}$ & 12 month & $\mathrm{R}$ & $\begin{array}{l}\text { Client educa- } \\
\text { tion and behav- } \\
\text { ior change }\end{array}$ & Service delivery \\
\hline Chen [54] & Rural & $\begin{array}{l}\text { Infectious disease } \\
\text {-schistosomiasis }\end{array}$ & $\begin{array}{l}501 \text { healthy } \\
\text { residents }\end{array}$ & $\begin{array}{l}\text { SMS-based health pro- } \\
\text { motion for schistosomi- } \\
\text { asis prevention }\end{array}$ & 10 month & $\mathrm{R}$ & $\begin{array}{l}\text { Client educa- } \\
\text { tion and behav- } \\
\text { ior change }\end{array}$ & Service delivery \\
\hline
\end{tabular}




\begin{tabular}{|c|c|c|c|c|c|c|c|c|}
\hline Author & Setting & Disease area & $\begin{array}{l}\text { Population } \\
\text { (n) }\end{array}$ & Study description & & $\begin{array}{l}\text { Type of } \\
\text { device }\end{array}$ & $\begin{array}{l}\text { mHealth } \\
\text { domain }\end{array}$ & $\begin{array}{l}\text { Health system } \\
\text { domain }\end{array}$ \\
\hline Chen [48] & Urban & $\begin{array}{l}\text { Maternal and child } \\
\text { health }\end{array}$ & $\begin{array}{l}180 \text { children } \\
\text { with allergic } \\
\text { rhinitis }\end{array}$ & $\begin{array}{l}\text { SMS-based health edu- } \\
\text { cation for allergic rhini- } \\
\text { tis management }\end{array}$ & 12 month & $\mathrm{R}$ & $\begin{array}{l}\text { Client educa- } \\
\text { tion and behav- } \\
\text { ior change }\end{array}$ & Service delivery \\
\hline $\mathrm{Xu}[41]$ & Urban & $\begin{array}{l}\text { Infectious disease } \\
\text {-HIV }\end{array}$ & $\begin{array}{l}71 \text { HIV pa- } \\
\text { tients }\end{array}$ & $\begin{array}{l}\text { SMS-based medication } \\
\text { adherence intervention }\end{array}$ & 12 month & $\mathrm{R}$ & $\begin{array}{l}\text { Client educa- } \\
\text { tion and behav- } \\
\text { ior change }\end{array}$ & Service delivery \\
\hline $\mathrm{Ni}[36]$ & Urban & $\begin{array}{l}\text { Maternal and child } \\
\text { health }\end{array}$ & $\begin{array}{l}460 \text { pregnant } \\
\text { women }\end{array}$ & $\begin{array}{l}\text { SMS-based health edu- } \\
\text { cation }\end{array}$ & 5 month & $\mathrm{R}$ & $\begin{array}{l}\text { Client educa- } \\
\text { tion and behav- } \\
\text { ior change }\end{array}$ & Service delivery \\
\hline Liu [37] & Urban & $\begin{array}{l}\text { Noncommunicable } \\
\text { disease } \\
\text {-acute coronary syn- } \\
\text { drome }\end{array}$ & $\begin{array}{l}82 \mathrm{ACS}^{\mathrm{e}} \text { pa- } \\
\text { tients }\end{array}$ & $\begin{array}{l}\text { SMS based medication } \\
\text { adherence intervention }\end{array}$ & 1 month & $\mathrm{R}$ & $\begin{array}{l}\text { Client educa- } \\
\text { tion and behav- } \\
\text { ior change }\end{array}$ & Service delivery \\
\hline Zhou [55] & Rural & $\begin{array}{l}\text { Maternal and child } \\
\text { health }\end{array}$ & $\begin{array}{l}\text { N250 preg- } \\
\text { nant women }\end{array}$ & $\begin{array}{l}\text { SMS-based health edu- } \\
\text { cation for HIV preven- } \\
\text { tion }\end{array}$ & 1 month & $\mathrm{R}$ & $\begin{array}{l}\text { Client educa- } \\
\text { tion and behav- } \\
\text { ior change }\end{array}$ & Service delivery \\
\hline $\mathrm{He}[50]$ & Urban & $\begin{array}{l}\text { Others } \\
\text {-general health }\end{array}$ & $\begin{array}{l}100 \text { residents } \\
\text { with smart- } \\
\text { phone }\end{array}$ & $\begin{array}{l}\text { Smartphone-based pe- } \\
\text { dometer "app" }\end{array}$ & 6 months & $\mathrm{S}$ & $\begin{array}{l}\text { Sensors and } \\
\text { point-of-care } \\
\text { diagnosis }\end{array}$ & Service delivery \\
\hline
\end{tabular}

${ }^{\mathrm{a}}$ PDA: personal digital assistant.

${ }^{\mathrm{T}} \mathrm{TB}$ : tuberculosis.

${ }^{\mathrm{c}}$ HIV: human immunodeficiency virus.

${ }^{\mathrm{d}}$ CVD: cardiovascular disease.

${ }^{\mathrm{e}} \mathrm{ACS}$ : acute coronary syndrome.

${ }^{\wedge} \mathrm{R}$ : regular mobile phone.

*S: smartphone.

The search of registered clinical trials identified 23 additional mHealth registered RCTs (Multimedia Appendix 2). Although 12 of these studies were listed as completed, we were only able to find 5 studies with published results. All 5 studies were identified during the original systematic review of the literature $[29,32,51,52,65]$. Consistent with the published RCTs, the majority of the interventions described in the registry focused on client education and behavior change using simple text messaging.

\section{Role of mHealth in the Health System}

Applying the adapted health system framework (Table 2), we found the client education and behavioral change communication was the most commonly targeted mHealth domain $(n=32)$ $[18,19,21,25,29-49,51,54-57,63,65]$. It was found that 5 interventions addressed sensors and point-of-care diagnostics $[22,24,26,50,66], 5$ interventions focused on data collection and reporting [23,27,28,60,61], 3 interventions involved registries and vital events tracking $[59,62,64], 2$ interventions focused on electronic decision support [52,58], 1 intervention involved electronic health records [20], and 1 intervention delivered provider training and education [53]. There were no interventions identified in the domains of provider to provider training, provider work planning and scheduling, human resources management, supply chain management, or financial transactions and incentives. From a health systems perspective, most studies targeted service delivery $(\mathrm{n}=38)$ $[18,19,21,23-26,29-52,54-57,63,65,66]$. Few interventions focused on the provision or management of information $(n=2)$ [60,61], health workforce support $(n=1)$ [53], medicines and technologies $(n=3)[22,27,28]$, or leadership and governance $(n=5)[20,58,59,62,64]$.

\section{Risk of Bias Assessment}

For the RCTs, risk of bias was mostly classified as either low or unclear (Table 3). Four studies did not provide sufficient information to assess risk $[34,35,43,47]$. 
Table 2. Health system framework assessment of the mHealth interventions.

\begin{tabular}{|c|c|c|c|c|c|c|c|c|}
\hline \multirow[t]{2}{*}{ mHealth Functionality } & \multicolumn{3}{|c|}{ Health System Structural Component } & \multirow[b]{2}{*}{$\begin{array}{l}\text { Health } \\
\text { Workforce }\end{array}$} & \multirow[b]{2}{*}{$\begin{array}{l}\text { Medicines/ } \\
\text { Technolo- } \\
\text { gies }\end{array}$} & \multirow[b]{2}{*}{$\begin{array}{l}\text { Informa- } \\
\text { tion }\end{array}$} & \multirow[b]{2}{*}{$\begin{array}{l}\text { Service De- } \\
\text { livery }\end{array}$} & \multirow[b]{2}{*}{ Sub-total } \\
\hline & $\begin{array}{l}\text { Leadership/ } \\
\text { Governance }\end{array}$ & Financing & Payment & & & & & \\
\hline Education/behavioral & & & & & & & 32 & 32 \\
\hline Sensors/point-of-care devices & & & & & 1 & & 4 & 5 \\
\hline Registries/vital events tracking & 3 & & & & & & & 3 \\
\hline Data collection and reporting & & & & & 2 & 2 & 1 & 5 \\
\hline Electronic health records & 1 & & & & & & & 1 \\
\hline Electronic decision support & 1 & & & & & & 1 & 2 \\
\hline \multicolumn{9}{|c|}{ Provider to provider communication } \\
\hline Provider training/education & & & & 1 & & & & 1 \\
\hline \multicolumn{9}{|l|}{ Human resources management } \\
\hline \multicolumn{9}{|l|}{ Supply chain management } \\
\hline \multicolumn{9}{|l|}{ Financial transactions/incentives } \\
\hline Sub-total & 5 & & & 1 & 3 & 2 & 38 & \\
\hline
\end{tabular}

Table 3. Risk of bias assessment for randomized controlled trials.

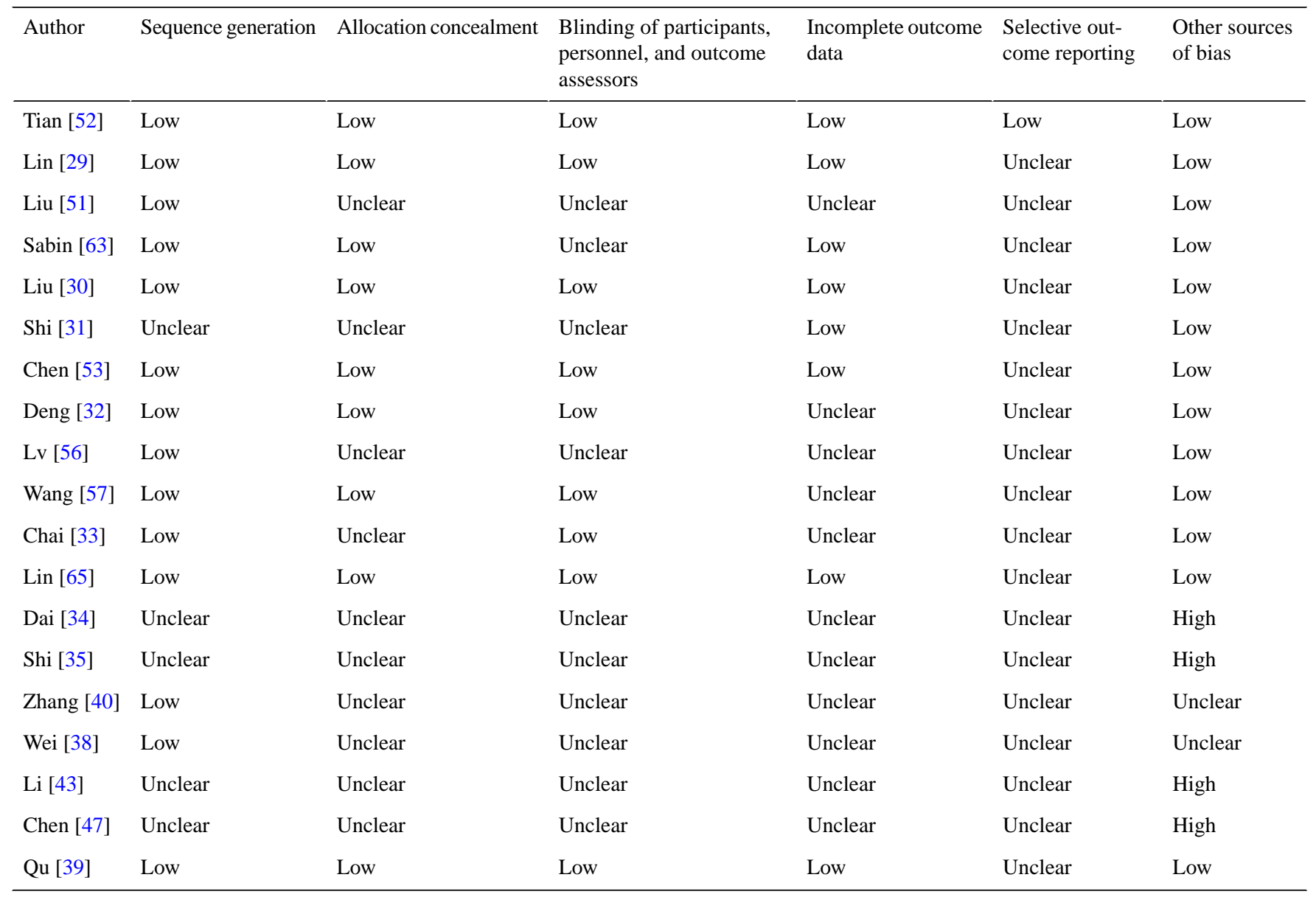




\section{Discussion}

\section{Principal Findings}

In this study, we reviewed studies and registered trials for studies published in the peer-reviewed journals involving mHealth interventions in China. We particularly focused on the extent to which mHealth interventions had the capacity to contribute to health care strengthening in the context of a rapidly evolving disease burden. Although we did observe an increasing focus on NCDs, there was little evidence of the development of mHealth interventions that were likely to substantially strengthen health care systems. We also noted a large disparity in the development of mHealth interventions that were focused on rural as opposed to urban areas. In addition, the quality of evidence provided in relation to effectiveness of such interventions is generally poor.

\section{Comparison With Other Reviews}

Beratarrechea et al [11] conducted a review to examine the role of mHealth intervention on the management of NCDs in LMICs, with a focus on the use of SMS and automated voice interventions. The study found that there were significant improvement on certain clinical outcomes and processes of care. Peiris et al further performed a review to explore the impact of all mHealth interventions on health care quality for NCDs in LMICs. Similar to our findings, there were few high-quality studies, and most of the studies used the SMS for patient behavior change. Very few studies addressed the mHealth intervention as a health system strengthening tool.

\section{Health System Strengthening}

On the basis of the literature we have identified, the development of mHealth interventions by academia in China remains relatively under-developed, in terms of both scope and capability. Interventions mostly utilized a texting tool to provide client education and behavior change. We identified a focus on only 7 of the 12 mHealth domains, with no interventions concentrating on interprovider communication or health service management, including financial transactions. In addition, all the interventions were developed as stand-alone tools to deliver health services, with little or no exploration of how integration within existing or developing health systems can be achieved.

\section{Health Equality}

Equitable access to quality health services is an important dimension of an effective health system. In China, around 50\% of the population is based in rural regions, where health outcomes are, in general, poorer than those among urban communities. Addressing such inequities is a public health priority, and mHealth strategies may provide a particular opportunity to reduce gaps that relate to weaker health systems. As China's mobile network reaches far and deep into its rural areas, mHealth solutions provide a real opportunity to strengthen rural health systems. Despite the huge potentials of mHealth help in closing the health equity gap, few academic studies in China has chosen to focus on this area. The regional imbalance identified in this review may be explained by the greater convenience of conducting studies in urban communities. However, the potential for mHealth to impact on health outcome inequities cannot be addressed if the digital gulf between those who have access to mobile technology in urban areas and those who do not have access in rural areas is not reduced. Similar considerations are relevant to other disadvantaged subgroups of population, including those with relatively low literacy or socioeconomic status.

\section{Quality of Evidence}

A key objective of mHealth research should be to provide useful and reliable evidence for end users, including policy-makers in the context of those innovations aimed at improving health outcomes through deployment in the public health care system. Our review found that published and planned mHealth studies in China largely have not and will not produce such outcomes. Fewer than $40 \%$ of the published studies utilized an RCT design and all were of uncertain or poor quality based on objective measures. The majority of the reports were descriptive, with no apparent attempt to determine efficacy or effectiveness. Study outcomes were largely the product of low-quality and small-scale experiments, which provided little understanding of the true impact of an intervention with large-scale real-world implementation within complex health systems.

\section{Limitations}

There are several limitations to this review. Firstly, we were not able to conduct a quantitative meta-analysis of the outcomes due to the heterogeneity of the RCTs. We identified a number of ongoing trials from the trial registry. The published results of those trials will enable to provide increased power to determine the size of the effect of mHealth interventions on health outcomes. Second, although the adapted health system framework was useful to evaluate the mHealth intervention as a health system strengthening tool, a single study may address multiple mHealth domains or health system domains. We only reported the primary functionality of the mHealth intervention and the key aspect that the intervention addressed in the health system. Finally, this review mainly targeted academic studies in the literature. We should note that China is experiencing rapid development in mHealth technology in the commercial world, many of which may have health system implications that we had limited ability to evaluate in this review.

\section{Conclusions}

mHealth has the potential to overcome some of the challenges due to the rapid changing environment of health care needs and provision in China. However, this potential can only be realized through the continual development of mHealth interventions to strengthen the health system, utilizing a subsequent rigorous approach to generating high-quality evidence about the likely implications of "real world implementation." Therefore, we outline three recommendations for future mHealth research and development in China: (1) mHealth studies should not be conducted as the standalone technical study evaluating its efficacy in the vacuum of the social context, (2) promote the development of integrated mHealth interventions as a tool to serve the existing health system, (3) focus on developing and evaluating mHealth interventions with the potential to reduce health outcome disparities within the population, and (4) conduct large-scale rigorously designed "real world" evaluation of 
mHealth interventions focused on health system strengthening. priority.

Specific public and private investment into such research is a

\section{Acknowledgments}

We thank the support from Dr Puhong Zhang, the Acting Director of the China Center for mHealth Innovation, and the funding support from Qualcomm Wireless Reach.

\section{Conflicts of Interest}

None declared.

\section{Multimedia Appendix 1}

Detailed search strategy for each database used.

[PDF File (Adobe PDF File), 27KB-Multimedia Appendix 1]

\section{Multimedia Appendix 2}

Table: Registered randomized controlled trials in clinical trials database.

[PDF File (Adobe PDF File), 27KB-Multimedia Appendix 2]

\section{References}

1. Yusuf S, Reddy S, Ounpuu S, Anand S. Global burden of cardiovascular diseases: part I: general considerations, the epidemiologic transition, risk factors, and impact of urbanization. Circulation 2001 Nov 27;104(22):2746-2753 [FREE Full text] [Medline: 11723030$]$

2. Yang G, Kong L, Zhao W, Wan X, Zhai Y, Chen LC, et al. Emergence of chronic non-communicable diseases in China. Lancet 2008 Nov 08;372(9650):1697-1705. [doi: 10.1016/S0140-6736(08)61366-5] [Medline: 18930526]

3. Wang L, Kong L, Wu F, Bai Y, Burton R. Preventing chronic diseases in China. Lancet 2005 Nov 19;366(9499):1821-1824. [doi: 10.1016/S0140-6736(05)67344-8] [Medline: 16298221]

4. Yip WC, Hsiao WC, Chen W, Hu S, Ma J, Maynard A. Early appraisal of China's huge and complex health-care reforms. Lancet 2012 Mar 03;379(9818):833-842. [doi: 10.1016/S0140-6736(11)61880-1] [Medline: 22386036]

5. Ramesh M, Wu X, He AJ. Health governance and healthcare reforms in China. Health Policy Plan 2014 Sep;29(6):663-672. [doi: 10.1093/heapol/czs109] [Medline: 23293100]

6. WHO. 2011. mHealth: New horizons for health through mobile technologies URL: http://www.who.int/goe/publications/ goe mhealth web.pdf [accessed 2017-03-03] [WebCite Cache ID 6ogKZD5HA]

7. MIIT. 2014. 2014 Communication Industry Statistical Bulletin URL: http://www.miit.gov.cn/n1146312/n1146904/n1648372/ c3337169/content.html [accessed 2017-03-03] [WebCite Cache ID 6ogKeyIsj]

8. NIELSEN. 2015. Chinese Smartphone Market Now Driven By Upgrading URL: http://www.nielsen.com/cn/en/press-room/ 2015/Nielsen-Chinese-Smartphone-Market-Now-Driven-by-Upgrading-EN.html [accessed 2017-03-03] [WebCite Cache ID $60 \mathrm{gKkqpyR}]$

9. $\quad$ Everett R. Diffusion of Innovations, 5th Edition. New York: Free Press; Aug 16, 2003.

10. Peiris D, Praveen D, Johnson C, Mogulluru K. Use of mHealth systems and tools for non-communicable diseases in lowand middle-income countries: a systematic review. J Cardiovasc Transl Res 2014 Nov;7(8):677-691. [doi: 10.1007/s12265-014-9581-5] [Medline: 25209729]

11. Beratarrechea A, Lee AG, Willner JM, Jahangir E, Ciapponi A, Rubinstein A. The impact of mobile health interventions on chronic disease outcomes in developing countries: a systematic review. Telemed J E Health 2014 Jan;20(1):75-82 [FRE Full text] [doi: 10.1089/tmj.2012.0328] [Medline: 24205809]

12. Goel S, Bhatnagar N, Sharma D, Singh A. Bridging the human resource gap in primary health care delivery systems of developing countries with mHealth: narrative literature review. JMIR Mhealth Uhealth 2013 Dec 03;1(2):e25 [FREE Full text] [doi: 10.2196/mhealth.2688] [Medline: 25099436]

13. Moher D, Liberati A, Tetzlaff J, Altman DG. Preferred reporting items for systematic reviews and meta-analyses: the PRISMA statement. Br Med J 2009 Jul 21;339:b2535 [FREE Full text] [Medline: 19622551]

14. Labrique A, Vasudevan L, Kochi E, Fabricant R, Mehl G. mHealth innovations as health system strengthening tools: 12 common applications and a visual framework. Glob Health Sci Pract 2013 Aug;1(2):160-171 [FREE Full text] [doi: 10.9745/GHSP-D-13-00031] [Medline: 25276529]

15. Hsiao W. Xa.yimg. 2003. What is a Health System? Why Should We Care? URL: https://xa.yimg.com/kq/groups/20489237/ 996876100/name/William+Hsiao+-+What+is+Health+System+2003.pdf [accessed 2017-02-22] [WebCite Cache ID 6oSVoKmyB] 
16. WHO. 2007. Everybody's business: Strengthening health systems to improve health outcomes URL: http://www.who.int/ healthsystems/strategy/everybodys business.pdf [accessed 2017-03-03] [WebCite Cache ID 6ogLSWKK9]

17. Higgins J, Altman DG, Gøtzsche PC, Jüni P, Moher D, Oxman AD, Cochrane Bias Methods Group, Cochrane Statistical Methods Group. The Cochrane Collaboration's tool for assessing risk of bias in randomised trials. Br Med J 2011 Oct 18;343:d5928 [FREE Full text] [Medline: 22008217]

18. Deng X, Ye L, Wang Y, Zhu T. A cross-sectional study to assess the feasibility of a short message service to improve adherence of outpatients undergoing sedation gastrointestinal endoscopy in the People's Republic of China. Patient Prefer Adherence 2014;8:1293-1297 [FREE Full text] [doi: 10.2147/PPA.S68814] [Medline: 25278748]

19. Chen H, Mishara BL, Liu XX. A pilot study of mobile telephone message interventions with suicide attempters in China. Crisis 2010;31(2):109-112. [doi: 10.1027/0227-5910/a000017] [Medline: 20418217]

20. Zhao J, Zhang Z, Guo H, Li Y, Xue W, Ren L, et al. E-health-oriented community health information system in china: our challenges, solution, and experience. Telemed J E Health 2011 Sep;17(7):584-588. [doi: 10.1089/tmj.2011.0024] [Medline: 21749261]

21. Mao Y, Zhang Y, Zhai S. Mobile phone text messaging for pharmaceutical care in a hospital in China. J Telemed Telecare 2008;14(8):410-414. [doi: 10.1258/jtt.2008.080406] [Medline: 19047450]

22. Qiao J, Xu L, Zhu Z, Zhu F, Liu Z, Qian B, et al. Inter- and intraobserver reliability assessment of the axial trunk rotation: manual versus smartphone-aided measurement tools. BMC Musculoskelet Disord 2014 Oct 11;15:343 [FREE Full text] [doi: 10.1186/1471-2474-15-343] [Medline: 25305758]

23. Guan Z, Wei B, Meng Z. [Development of remote wireless mobile voiding diary and a report of its objective voiding in 20 young people]. Beijing Da Xue Xue Bao 2010 Aug 18;42(4):476-479 [FREE Full text] [Medline: 20721269]

24. Ye Y, Wang J, Xie Y, Zhong J, Hu Y, Chen B, et al. Global teleophthalmology with iPhones for real-time slitlamp eye examination. Eye Contact Lens 2014 Sep;40(5):297-300. [doi: 10.1097/ICL.0000000000000051] [Medline: 25083779]

25. Yin L, Liu P, Meng Y, Guan B, Hu B, Chen S, et al. [Xue Ye Jing Hua Zhong Xin Yi Huan Zi Xun Sui Fang Hu Dong Ping Tai Zai Lin Chuang De Ying Yong]. Xian Dai Yu Fang Yi Xue 2009;36(4):781-782.

26. Yang L, Wang X, Liu X, Dong H. [Ying Yong Zhao Xiang Shou Ji Dui Mian Bu Cuo Chuang Yan Zhong Xing Jin Xing Yuan Cheng Fen Ji De Ke Xing Xing]. Zheng Zhou Da Xue Yi Xue Bao 2009(6):1229-1232.

27. Wang ZX, Zhang M, Wu J, Sun L, Jiang T, Song C. [Yi Zhong Xin De Ji Shi Xing Tu Xiang Fa Shan Shi Diao Cha Ji Shu He Xiao Guo Ping Jia]. Ying Yang Xue Bao 2014;3:288-295.

28. Smith L, Hua J, Seto E, Du S, Zang J, Zou S, et al. Development and validity of a 3-day smartphone assisted 24-hour recall to assess beverage consumption in a Chinese population: a randomized cross-over study. Asia Pac J Clin Nutr 2014;23(4):678-690 [FREE Full text] [Medline: 25516327]

29. Lin P, Wang Y, Levine E, Askew S, Lin S, Chang C, et al. A text messaging-assisted randomized lifestyle weight loss clinical trial among overweight adults in Beijing. Obesity (Silver Spring) 2014 May;22(5):E29-E37 [FREE Full text] [doi: 10.1002/oby.20686] [Medline: 24375969]

30. Liu Z, Chen S, Zhang G, Lin A. Mobile phone-based lifestyle intervention for reducing overall cardiovascular disease risk in Guangzhou, China: a pilot study. Int J Environ Res Public Health 2015 Dec 17;12(12):15993-16004 [FREE Full text] [doi: 10.3390/ijerph121215037] [Medline: 26694436]

31. Shi H, Jiang X, Yu C, Zhang Y. Use of mobile phone text messaging to deliver an individualized smoking behaviour intervention in Chinese adolescents. J Telemed Telecare 2013 Jul;19(5):282-287. [doi: 10.1177/1357633X13495489] [Medline: 24163238]

32. Deng X, Wang Y, Zhu T, Zhang W, Yin Y, Ye L. Short message service (SMS) can enhance compliance and reduce cancellations in a sedation gastrointestinal endoscopy center: a prospective randomized controlled trial. J Med Syst 2015 Jan;39(1):169. [doi: 10.1007/s10916-014-0169-z] [Medline: 25476268]

33. Chai SJ, Tan F, Ji Y, Wei X, Li R, Frost M. Community-level text messaging for 2009 H1N1 prevention in China. Am J Prev Med 2013 Aug;45(2):190-196. [doi: 10.1016/j.amepre.2013.03.014] [Medline: 23867026]

34. Dai MQ, Lou Q, Zhang Y. [Wu Xian Tong Xun Duan Xin Jiao Yu Zai Tang Niao Bing Huan Zhe Zi Wo Guan Li Zhong De Ying Yong]. Zhong Hua Hu Li Za Zhi 2010;3:225-227.

35. Shi HJ, Yu C, Zhang S, Zhang Y, An A, Tan H, et al. [Qing Shao Nian Jie Yan Xin Yi Tong Xin Ji SHu Gan Yu Shi Shi Fang An Ke Xing Xing Fen Xi]. Zhong Guo Xue Xiao Wei Sheng 2010;12:1434-1432.

36. Ni YX, Pan J, Xing Z, Bi X, Dong Y, Cong X. [Duan Xin Qun Fa Ping Tai Fu Zhu Yun Fu Xue Xiao Jian Kang Jiao Yu De Xiao Guo Guan Cha]. Zhong Hua Hu Li Za Zhi 2013;12:1093-1095.

37. Liu YS, Li Y, Wu Y. [Duan Xin Jiao Yu Gai Shan Ji Xing Guan Mai Zong He Zheng Huan Zhe Jie Ru Shu Hou Fu Yao Yi Cong Xing De Xiao Guo Yan Jiu]. Zhong Hua Hu Li Za Zhi 2015;06:660-665.

38. Wei N, Chen W, Zhang X, Mo Y, Lei H, Wang J, et al. [Duan Xin Ping Tai Jiao Yu Zai Ti Gao Man Xing Shen Zang Huan Zhe Fu Yao Yi Cong Xing Zhong De Ying Yong Yan Jiu]. Zhong Guo Quan Ke Yi Xue 2013;25:2287-2289.

39. Qu JL, Zhang T, Song J, Fang H. [Chu Yuan Hou Jing Shen Fen Lie Zheng Huan Zhe Fu Yao Yi Cong Xing Dian Hua He Duan Xin Gan Yu De Yi Nian Sui Fang]. Zhong Guo Xin Li Wei Sheng Za Zhi 2015;09:656-661.

40. Zhang J, Wu Y, Yuan M, Yin Y, Wang W, Zhang L, et al. [Qi Xiang Huan Jing Yu Jing Yu Bao Ji Shu Dui Xiao Chuan Er Tong Sheng Ming Zhi Liang Gan Yu De Yan Jiu]. Zhong Guo Shi Yong Er Ke Za Zhi 2013;03:189-194. 
41. Fang X, Shen G, Wu L, Chen Y, Xu W, Hu S. [An Hui Zhong Bu Zhi Ye Ren Qun Gao Xue Ya Jiao Yu Gan Yu Xiao Guo Ping Jia]. Zhong Hua Gao Xue Ya Za Zhi 2010;10:965-969.

42. Fang X, Shen G, Wu L, Chen Y, Xu W, Hu S. [An Hui Zhong Bu Zhi Ye Ren Qun Gao Xue Ya Jiao Yu Gan Yu Xiao Guo Ping Jia]. Zhong Hua Gao Xue Ya Za Zhi 2010;10:965-969.

43. Li LX, Wu K, Huang L, Xu H, Luo M. [Duan Xin Jiao Yu Dui Yun Fu Shan Shi Ji Ti Li Huo Dong Xing Wei De Ying Xiang]. Guang Dong Yi Xue 2014;24:3927-3929.

44. Qing XY, Shao Q, Nie J, Wang L, Ma H, Hu Y. [Duan Xin Jiao Yu Dui Xue Ye Tou Xi Qi Jian Huan Zhe Ti Zhi Liang De Ying Xiang]. Zhong Hua Hu Li Za Zhi 2012;04:311-313.

45. Xie B, Ju C, Sun Z, Han J, Liu L. [Tang Niao Bing Zi Wo Guan Li Pei Xun Zhi Dao Wang Luo Dui Er Xing Tang Niao Bing Huan Zhe Zi Wo Xiao Neng De Ying Xiang Yan Jiu]. Zhong Guo Quan Ke Yi Xue 2012;31:3616-3612.

46. Zhao M, Yang L, Chen Q, Zhai S. [Tang Niao Bing Huan Zhe Ge Ti Hua Shou Ji Duan Xin Yao Xue Fu Wu De Xiao Guo Yan Jiu]. Zhong Guo Yao Fang 2010;42:4020-4023.

47. Chen J, Zhang S, Wang X. [Bei Jing Shi Mou She Qu Si Ge Yue Nei Mu Ru Wei Yang Gan Yu Yan Jiu]. Zhong Guo Er Tong Bao Jian Za Zhi 2014;05:469-466.

48. Chen J, Li Y, Mou Z, He S, Peng L. [Huan Jing Yu Jiang Kang Duan Xin Yu Jing Dui Er Tong Bian Ying Xing Bi Yan Liao Xiao De Ying Xiang]. Lin Chuang Er Bi Yan Hou Tou Jing Wai Ke Za Zhi 2015;03:218-214.

49. Jiang H, Li M, Wen LM, Hu Q, Yang D, He G, et al. Effect of short message service on infant feeding practice: findings from a community-based study in Shanghai, China. JAMA Pediatr 2014 May;168(5):471-478. [doi: 10.1001/jamapediatrics.2014.58] [Medline: 24639004]

50. He JEA. [Zhi Neng Shou Ji Jian Kang Guang Li Ruan Jian Dui She Qu Ren Qun Jian Kang Shui Ping He Sheng Huo Zhi Liang De Gan Yu Xiao Guo Yan Jiu]. Zhong Guo Quan Ke Yi Xue 2015;19:2333-2336.

51. Liu X, Lewis JJ, Zhang H, Lu W, Zhang S, Zheng G, et al. Effectiveness of electronic reminders to improve medication adherence in tuberculosis patients: a cluster-randomised trial. PLoS Med 2015 Sep;12(9):e1001876 [FREE Full text] [doi: 10.1371/journal.pmed.1001876] [Medline: 26372470]

52. Tian M, Ajay VS, Dunzhu D, Hameed SS, Li X, Liu Z, et al. A cluster-randomized, controlled trial of a simplified multifaceted management program for individuals at high cardiovascular risk (SimCard Trial) in rural Tibet, China, and Haryana, India. Circulation 2015 Sep 01;132(9):815-824 [FREE Full text] [doi: 10.1161/CIRCULATIONAHA.115.015373] [Medline: 26187183]

53. Chen Y. Use of text messages to communicate clinical recommendations to health workers in rural China: a cluster-randomized trial. Bull World Health Organ 2014;92(7):474-481. [doi: 10.2471/BLT.13.127076]

54. Chen LEA. [Shou Ji Gan Yu Mo Shi Zai Liu Dong Ren Kou Xue Fang Gong Zuo Zhong De Zuo Yong]. Xian Dai Yu Fang Yi Xue 2013;09:1754-1756.

55. Zhou REA. [Guang Xi Wu Yu Zhen Yun Fu Yu Fang Ai Zi Bing Mu Ying Chuan Bo Jian Kang Jiao Yu Mo Shi Yan Jiu Yu Shi Jian]. Zhong Guo Fu You Bao Jian 2015;24:4103-4107.

56. Lv Y, Zhao H, Liang Z, Dong H, Liu L, Zhang D, et al. A mobile phone short message service improves perceived control of asthma: a randomized controlled trial. Telemed J E Health 2012;18(6):420-426. [doi: 10.1089/tmj.2011.0218] [Medline: 22667695]

57. Wang K, Wang C, Xi L, Zhang Y, Ouyang Y, Lou H, et al. A randomized controlled trial to assess adherence to allergic rhinitis treatment following a daily short message service (SMS) via the mobile phone. Int Arch Allergy Immunol 2014;163(1):51-58. [doi: 10.1159/000356317] [Medline: 24248037]

58. Li Y, Fang L, Gao S, Wang Z, Gao H, Liu P, et al. Decision support system for the response to infectious disease emergencies based on WebGIS and mobile services in China. PLoS One 2013;8(1):e54842 [FREE Full text] [doi: 10.1371/journal.pone.0054842] [Medline: 23372780]

59. Li Z. Hand, foot and mouth disease in Chinavaluating an automated system for the detection of outbreaks. Bull World Health Organ 2014;92(9):656-663. [doi: 10.2471/BLT.13.130666]

60. Guo Y, Su XM. Mobile device-based reporting system for Sichuan earthquake-affected areas infectious disease reporting in China. Biomed Environ Sci 2012 Dec;25(6):724-729 [ [FREE Full text] [doi: 10.3967/0895-3988.2012.06.016] [Medline: 23228844]

61. Yang C, Yang J, Luo X, Gong P. Use of mobile phones in an emergency reporting system for infectious disease surveillance after the Sichuan earthquake in China. Bull World Health Organ 2009 Aug;87(8):619-623 [FREE Full text] [Medline: $\underline{19705013]}$

62. Ma J, Zhou M, Li Y, Guo Y, Su X, Qi X, et al. Design and application of the emergency response mobile phone-based information system for infectious disease reporting in the Wenchuan earthquake zone. J Evid Based Med 2009 May;2(2):115-121. [doi: 10.1111/j.1756-5391.2009.01018.x] [Medline: 21348998]

63. Sabin LL, Bachman DM, Gill CJ, Zhong L, Vian T, Xie W, et al. Improving adherence to antiretroviral therapy with triggered real-time text message reminders: the China adherence through technology study. J Acquir Immune Defic Syndr 2015 Aug 15;69(5):551-559 [FREE Full text] [doi: 10.1097/QAI.0000000000000651] [Medline: 25886927] 
64. Zhang Y, Huan H, Chen Z, Zhang Q, Wu R, Jiang J, et al. [Construction of platform of Schistosoma japonicum infection real-time monitoring and early warning along Yangtze River]. Zhongguo Xue Xi Chong Bing Fang Zhi Za Zhi 2013 Dec;25(6):581-584. [Medline: 24490389]

65. Lin H, Chen W, Luo L, Congdon N, Zhang X, Zhong X, et al. Effectiveness of a short message reminder in increasing compliance with pediatric cataract treatment: a randomized trial. Ophthalmology 2012 Dec;119(12):2463-2470. [doi: 10.1016/j.ophtha.2012.06.046] [Medline: 22921386]

66. Yu Y, Li J, Liu J. M-HELP: a miniaturized total health examination system launched on a mobile phone platform. Telemed J E Health 2013 Nov;19(11):857-865. [doi: 10.1089/tmj.2013.0031] [Medline: 24050610]

\author{
Abbreviations \\ ACS: acute coronary syndrome \\ CHICTR: Chinese Clinical Trial Registry \\ CNKI: China National Knowledge of Infrastructure \\ COPD: chronic obstructive pulmonary disease \\ CVD: cardiovascular disease \\ HIV: human immunodeficiency virus \\ LMICs: low- and middle-income countries \\ NCDs: noncommunicable diseases \\ PDA: personal digital assistant \\ PRISMA: Preferred Reporting Items for Systematic Reviews and Meta-Analyses \\ RCTs: randomized controlled trials \\ SGIE: sedation gastrointestinal endoscopy \\ SMS: short message service \\ TB: tuberculosis \\ WHO: World Health Organization
}

Edited by G Eysenbach; submitted 26.10.16; peer-reviewed by J Apolinário-Hagen, $Y$ Wu; comments to author 29.12.16; revised version received 26.01.17; accepted 10.02.17; published 16.03.17

Please cite as:

Tian M, Zhang J, Luo R, Chen S, Petrovic D, Redfern J, Xu DR, Patel A

mHealth Interventions for Health System Strengthening in China: A Systematic Review

JMIR Mhealth Uhealth 2017;5(3):e32

URL: http://mhealth.jmir.org/2017/3/e32/

doi: $10.2196 /$ mhealth. 6889

PMID: 28302597

CMaoyi Tian, Jing Zhang, Rong Luo, Shi Chen, Djordje Petrovic, Julie Redfern, Dong Roman Xu, Anushka Patel. Originally published in JMIR Mhealth and Uhealth (http://mhealth.jmir.org), 16.03.2017. This is an open-access article distributed under the terms of the Creative Commons Attribution License (http://creativecommons.org/licenses/by/2.0/), which permits unrestricted use, distribution, and reproduction in any medium, provided the original work, first published in JMIR mhealth and uhealth, is properly cited. The complete bibliographic information, a link to the original publication on http://mhealth.jmir.org/, as well as this copyright and license information must be included. 